\title{
FATORES DA QUALIDADE DO SERVIÇO QUE AFETAM A FIDELIDADE DOS ALUNOS E SUAS INTENÇÕES EM RECOMENDAR A ESCOLA: UM ESTUDO NO SETOR DA EDUCAÇÃO PÚBLICA
}

\section{Factors of service quality that affect student loyalty and their intentions to recommend school: a study in the public education sector}

\section{Evadio Pereira Filho}

Doutorando do Programa de Pós-Graduação em Administração da Universidade Federal do Rio Grande do Norte (PPGA/UFRN). Professor do Instituto Federal de Educação, Ciência e Tecnologia da Paraíba (IFPB).email:evadio.filho@gmail.com

\section{Miguel Eduardo Moreno Añez}

Pós-Doutor em Administração Internacional pela École des Hautes Études Commerciales de Montréal, Canadá. Professor do Programa de Pós-Graduação em Administração da Universidade Federal do Rio Grande do Norte (PPGA/UFRN). email: anez1957@yahoo.com.br

\section{RESUMO}

Este estudo desenvolveu um ensaio teórico-empírico com 1550 alunos do ensino médio de escolas públicas paraibanas. O objetivo foi avaliar o impacto da qualidade do serviço educacional sobre intenções em recomendar a terceiros e fidelidade dos discentes, bem como em que medida esses efeitos se diferem em relação aos fatores demográficos e motivacionais. Foram apreendidas as expectativas do alunado e suas percepções de desempenho em relação a 18 atributos. Capturou-se ainda o quão propensos estavam em rematricular-se e em recomendar a escola. Ao empregar regressão linear múltipla, verificou-se que quatro e três atributos, respectivamente, apresentaram efeito significativo sobre fidelidade dos consumidores e intenção em recomendar a organização. Atendimento do pessoal administrativo e imagem corporativa constituem-se pontos de interseção, impactando positivamente nas duas variáveis comportamentais. Heterogeneidades no impacto da qualidade do serviço sobre fidelidade e intenções de recomendar foram percebidas nos agrupamentos de faixa etária, renda familiar, motivação e gênero.

Palavras-chave: Qualidade do serviço. Fidelidade. Intenções em recomendar. Ensino público.

\section{ABSTRACT}

This study developed a theoretical-empirical essay with 1550 high school students from public schools in the Paraíba. The objective was to evaluate the impact of quality of the educational service about intentions in recommending to third parties and fidelity of the students, as well as to what extent these effects differ in relation to the demographic and motivational factors. The expectations of the students and his performance perceptions in relation to 18 attributes were apprehended. It was also captured how prone they were to to enroll again and to recommend school. When using multiple linear regression, it was found that four and three attributes, respectively, had a significant effect on consumer loyalty and intention to recommend the organization. Attendance of the administrative staff and corporate image are points of intersection, positively impacting the two behavioral variables. Heterogeneities in the impact of quality of service about fidelity and intentions to recommend were perceived in the groupings of age, family income, motivation and gender.

Keywords: Service quality. Loyalty. Intentions to recommend. Public education. 


\section{INTRODUÇÃO}

O mercado do ensino, ano a ano, amplia a sua oferta. Entre 2008 e 2016, o quantitativo de escolas brasileiras que atuam no ensino médio passou de 25,3 para 28,3 mil estabelecimentos. Dentre estes, cerca de 20,1 mil pertencem ao sistema público. Em 2016, foram efetuadas 8,1 milhões de matrículas no ensino médio, o que representa um aumento de aproximadamente $0,7 \%$ em relação a 2015 . Deste montante, $87,5 \%$ foram executadas pela rede pública (INEP, 2017).

Os dados indicam que o segmento da educação pública é marcado por uma intensa competição. As escolas disputam alunos entre si para se manterem ativas no sistema de ensino e disporem de parcela maior de recursos financeiros. Nesse cenário, é essencial que a organização formule e implemente estratégias que possibilitem o desenvolvimento de diferenciais frente à concorrência. Para Sweis et al. (2016), o sucesso das instituições de ensino perpassa pela construção e manutenção de vantagens competitivas. E ofertar níveis de serviço condizentes com as expectativas dos consumidores passou a configurar-se como um fator chave, pois contribui para melhorar níveis de satisfação e favorece o boca-a-boca positivo (CARDONA; BRAVO, 2012; ALI et al., 2016; SWEIS et al., 2016).

Consoante Ramseook-Munhurrun e Nundlall (2013), os atuais gestores das instituições de ensino estão atribuindo uma maior atenção ao aperfeiçoamento dos pacotes de serviços ofertados. A procura por excelência passou a ser uma das principais preocupações dos administradores da área. A gerência precisa compreender os atributos do serviço que os clientes (em especial, os alunos) valorizam e, dentre estes, em quais a escola possui altos déficits de qualidade. Isso auxilia a estabelecer prioridades de ação, realocando recursos para aspectos mais relevantes (RAMSEOOK-MUNHURRUN; NUNDLALL, 2013; ALI et al., 2016). Ademais, Martínez-Argüelles e Batalla-Bisquets (2016) asseguram que o estudante fiel a uma organização tende a comprar com maior frequência e em maior volume, a ser mais resistente às ofertas de organizações rivais e a propagar positivamente a imagem da corporativa.

No mais, a compreensão do conceito da qualidade do serviço educacional não se restringe somente a análises singulares das discrepâncias, atributo a atributo, que existe entre as expectativas dos clientes (serviço esperado) e suas percepções de desempenho organizacional (serviço recebido). $\mathrm{Na}$ área do ensino, teóricos (VERGARA; QUESADA, 2011; CARDONA; BRAVO, 2012; ALI et al., 2016; MARTÍNEZ-ARGÜELLES; BATALLA-BISQUETS, 2016) notabilizam-se por investigações que tem como foco as relações entre qualidade do serviço e variáveis relativas ao comportamento do consumidor (por exemplo, satisfação e intenções de recompra).

A literatura realizou esforços para compreender os enlaces dessas variáveis no contexto da educação superior. Um conjunto de pesquisas evidenciaram o quanto a qualidade do serviço impactava direta e positivamente a satisfação dos clientes. Em geral, o aumento da qualidade percebida conduz a patamares crescentes de satisfação. Este fenômeno foi validado em diversos países, como Colômbia, Malásia e Espanha. Para esse fim, regressão linear múltipla e equações estruturais foram os métodos empregados com maior intensidade (VERGARA; QUESADA, 2011; CARDONA; BRAVO, 2012; ALI et al., 2016; MARTÍNEZ-ARGÜELLES; BATALLA-BISQUETS, 2016).

Em adição, houve divergências no que tange ao entendimento sobre a relação qualidade-lealdade. Pesquisadores defenderam a tese de que a qualidade do serviço exerceria efeitos diretos e positivos sobre a lealdade e sobre a disposição em recomendar (MARTÍNEZ-ARGÜELLES; BATALLA-BISQUETS, 2016). Outra tribo de autores discordava desse posicionamento. Para eles, a relação entre qualidade e fidelidade seria mediada pelo nível de satisfação. Logo, notava-se uma relação indireta entre as variáveis inicialmente citadas (VERGARA; QUESADA, 2011; ALI et al., 2016).

À luz do exposto, duas questões-chave passaram a nortear o presente estudo: a) como a qualidade do serviço ofertado por escolas públicas do ensino médio se relaciona com a fidelidade do discente e seu comportamento em recomendar a instituição?; b) em que medida os efeitos da qualidade do serviço sobre fidelidade e intenções em recomendar diferem dos fatores demográficos e motivacionais do aluno? Para esse fim, foram apreendidas as expectativas (importância) de 1550 alunos e suas percepções de desempenho em relação a um conjunto de 18 atributos, bem 
como as intenções de rematricular-se (fidelidade) e de recomendar a escola para outros. O objetivo é analisar o impacto da qualidade do serviço ofertado por vinte escolas públicas situadas no sertão paraibano sobre as variáveis comportamentais sobreditas, e como esse efeito distingue-se a partir de aspectos demográficos e motivacionais dos consumidores. Empregou-se a regressão linear múltipla, tendo como variáveis de controle a qualidade percebida em cada um dos atributos (escores de desempenho percebido subtraídos das expectativas). As variáveis explicadas foram intenções de rematricular-se na escola e de recomendá-la para outros. A posteriori, foram inclusas dummies, como variáveis independentes, a fim de avaliar possíveis diferenças nos efeitos intrínsecos ao relacionamento entre os construtos envolvidos.

O presente estudo respalda-se em recomendações de alguns teóricos. Ramseook-Munhurrun e Nundlall (2013) e Sweis et al. (2016) alegaram que há uma hegemonia de trabalhos empíricos que tratam sobre qualidade do serviço no segmento do ensino superior, sendo poucas as contribuições teóricas dentro do contexto educacional mais elementar. Já Ali et al. (2016) asseveram que é primordial uma discussão mais holística sobre o fenômeno da qualidade do serviço. Os autores recomendam uma melhor compreensão da qualidade do serviço em diversos contextos culturais, inclusive investigando como essa variável se relaciona com a satisfação e os comportamentos do consumidor. Por sua vez, Martínez-Argüelles e Batalla-Bisquets (2016) afirmam que os trabalhos que avaliam a correlação entre a qualidade percebida pelos estudantes e sua disposição em continuar estudando e o seu comportamento em recomendar a instituição de ensino, ainda estão em um estágio embrionário. Por conseguinte, esse estudo procura contribuir para minimizar estas lacunas acadêmicas, pois ampliou-se a discussão da relação causal entre os construtos sobreditos para uma categoria do ensino público ainda carente de ensaios empíricos.

Quanto às implicações gerenciais, este estudo fornece insights que auxiliam os gestores das escolas em suas decisões estratégicas. É possível verificar quais atributos do serviço mais impactam as intenções de recompra dos clientes e de seus comporta- mentos de indicação. Essas informações possibilitam que as escolas projetem de forma eficaz e eficiente a prestação do serviço, remanejando recursos para áreas que demandam ações prioritárias de melhorias. A gerência deve atenção especial aos atributos com elevada defasagem de qualidade e que apresentam impacto de alta magnitude sobre os comportamentos de intenções de rematrícula e de recomendar da organização.

\section{FUNDAMENTOS TEÓRICOS}

$\mathrm{Na}$ indústria do serviço, a qualidade é um diferencial competitivo (FITZSIMMONS; FITZSIMMONS, 2005). Para Grönroos (1984), a conformidade entre aquilo que o consumidor projeta receber (expectativas) e o que efetivamente recebe (desempenho percebido) simboliza a qualidade do serviço. A empresa que possui desempenho igual ou superior às expectativas de seus clientes, promove qualidade na prestação do serviço. Comportamentos organizacionais distintos acarretam insatisfação na clientela. É essencial que os gestores compreendam as discrepâncias entre o serviço esperado e recebido para que evitem a perda de negócios (GRÖNROOS, 1984).

Contudo, Ali et al. (2016) mencionam que a qualidade do serviço carrega traços particulares de contextos. Na educação, por exemplo, os autores avalizam que este conceito não se restringe somente aos padrões educacionais idealizados e julgados pelos alunos. Realçam que a terminologia engloba as mudanças significativas no próprio sistema educacional, na natureza das suas entradas, nos currículos, nas tecnologias educacionais, no ambiente socioeconômico, cultural e político na qual está inserida. A maioria dos educadores também incluem, na definição, o ambiente de aprendizagem (ALI et al., 2016). No mais, aspectos da intangibilidade e heterogeneidade, os quais cercam a concepção de serviço, alimentam a insegurança do consumidor (PARASURAMAN; ZEITHAML; BERRY, 1985). Por isso, a avaliação da qualidade do serviço perpassa pela delimitação de atributos operacionalizáveis que melhor representem o contexto pesquisado. 


\subsection{Qualidade em instituições de ensino}

A educação carece da adoção de ferramentas capazes de monitorar a qualidade e a satisfação dos clientes (RAMSEOOK-MUNHURRUN; NUNDLALL, 2013; ALI et al., 2016). Há uma miríade de estudos que visam contribuir com essa lacuna acadêmica e que enumeram atributos dos serviços educacionais.

Alguns literários (DESHIELDS JR. et al., 2005; NAVARRO et al., 2005; BUTTA; REHMAN, 2010; ALCÂNTARA et al., 2012) avaliaram o nível de satisfação de universitários com suas respectivas instituições de ensino. Constataram que know-how e atitude dos docentes, métodos de ensino aplicados e a coordenação do curso representam os elementos que mais atuam sobre a satisfação do aluno e que merecem maior atenção gerencial. Já outros teóricos (MOSTAFA, 2007; EBERLE, 2009; ANNAMDEVULA; SHEKHAR, 2012) investigaram sobre a robustez das cinco dimensões de qualidade propostas pelo SERVQUAL: tangibilidade (referente aos aspectos físicos), confiabilidade (cumprir o que foi prometido com exatidão), responsividade (capacidade de ajudar os usuários), garantia (segurança fornecida através das operações) e empatia (atenção individualizada ao usuário). Em geral, esse conjunto genérico de dimensões não foi sustentado, devido a uma diversidade maior ter sido identificada nos estudos, indicando tão somente que a qualidade é um construto multidimensional.

A Figura 1 ilustra os vinte e cinco trabalhos empíricos que serviram como fontes para identificar os atributos que melhor descrevem os serviços acadêmicos. A escolha dos Autores por esses estudos pauta-se nos seguintes critérios: contemporaneidade e adequação dos trabalhos ao recorte temático pesquisado, presença de variáveis que retratassem os serviços educacionais.
Figura 1 Inventário dos estudos que contêm atributos do serviço educacional

\begin{tabular}{|c|c|}
\hline Autor/Ano & País \\
\hline Deshields Jr. et al. (2005) & EUA \\
\hline Navarro et al. (2005) & Espanha \\
\hline Lorenço et al. (2006) & Brasil \\
\hline Abdullah (2006) & Malásia \\
\hline Mostafa (2007) & Egito \\
\hline Eberle (2009) & Brasil \\
\hline Brochado (2009) & Portugal \\
\hline Butta e Rehman (2010) & Paquistão \\
\hline Cunha et al. (2010) & Brasil \\
\hline Vergara e Quesada (2011) & Colômbia \\
\hline Campos et al. (2011) & Brasil \\
\hline Alcântara et al. (2012) & Brasil \\
\hline Annamdevula e Shekhar (2012) & Índia \\
\hline Khoo, Ha e Mcgregor (2017) & Singapura \\
\hline Cardona e Bravo (2012) & Colômbia \\
\hline Nyagowaa et al. (2013) & Quênia \\
\hline Milan et al. (2013) & Brasil \\
\hline Ramseook-Munhurrun e Nundlall (2013) & Maurícias \\
\hline Dos Santos (2014) & Brasil \\
\hline Souza et al. (2014) & Brasil \\
\hline Duarte e Piratelli (2015) & Brasil \\
\hline Lankara e Ye (2015) & Mianmar \\
\hline Ramos (2015) & Brasil \\
\hline Ali et al. (2016) & Malásia \\
\hline Ashraf et al. (2016) & Bangladesh \\
\hline Sweis et al., 2016 & Jordânia \\
\hline Pereira Filho, Tenório e Silva (2017) & Brasil \\
\hline
\end{tabular}

Fonte: Elaborada pelos autores 


\section{MÉTODO}

A Figura 2 ilustra o design metodológico adotado nesse estudo. De início, foi conduzida uma revisão de literatura, nacional e internacional, sobre a teoria da qualidade em serviços e os estudos empíricos em ambientes de ensino que abordavam essa temática. Nessa etapa, os objetivos foram mapear os atributos que simbolizam os serviços educacionais e eleger, dentre os instrumentos de pesquisa válidos, aquele que melhor atendesse aos critérios: a) contemplar o maior número dos atributos mais citados na literatura; b) mensurar a qualidade do serviço de modo mais apropriado ao setor público do ensino médio.

A ferramenta elaborada por Dos Santos (2014) foi escolhida. No entanto, alguns ajustes faziam-se necessários uma vez que o seu desenvolvimento foi originário do contexto de universidades privadas. $\mathrm{O}$ passo inicial foi confrontar as 33 variáveis embrionárias do instrumento selecionado com o rol de atributos mais citados na literatura. Esse filtro reduziu para 29 itens. $\mathrm{Na}$ sequência, submeteu-se essas variáveis ao crivo de 60 sujeitos (professores e alunos), sendo três examinadores de cada instituição de ensino, os quais foram selecionados por conveniência e acessibilidade. Estes executaram a função de juízes, analisando os níveis de clareza das sentenças que definiam cada variável, bem como opinaram sobre a irrelevância dos itens. Apontaram não só quais atributos deveriam ser excluídos por não fazer parte do contexto do ensino público, bem como propuseram certas reformulações nos termos usados a fim de adequar a linguagem ao cenário local. Ao final desse procedimento, dezoito variáveis relativas à qualidade do serviço compuseram o instrumento final.

Figura 2 Procedimentos metodológicos Fonte: Elaborada pelos autores

Revisão de Literatura sobre Gestão da Qualidade em Serviços e Qualidade em Instituições de Ensino

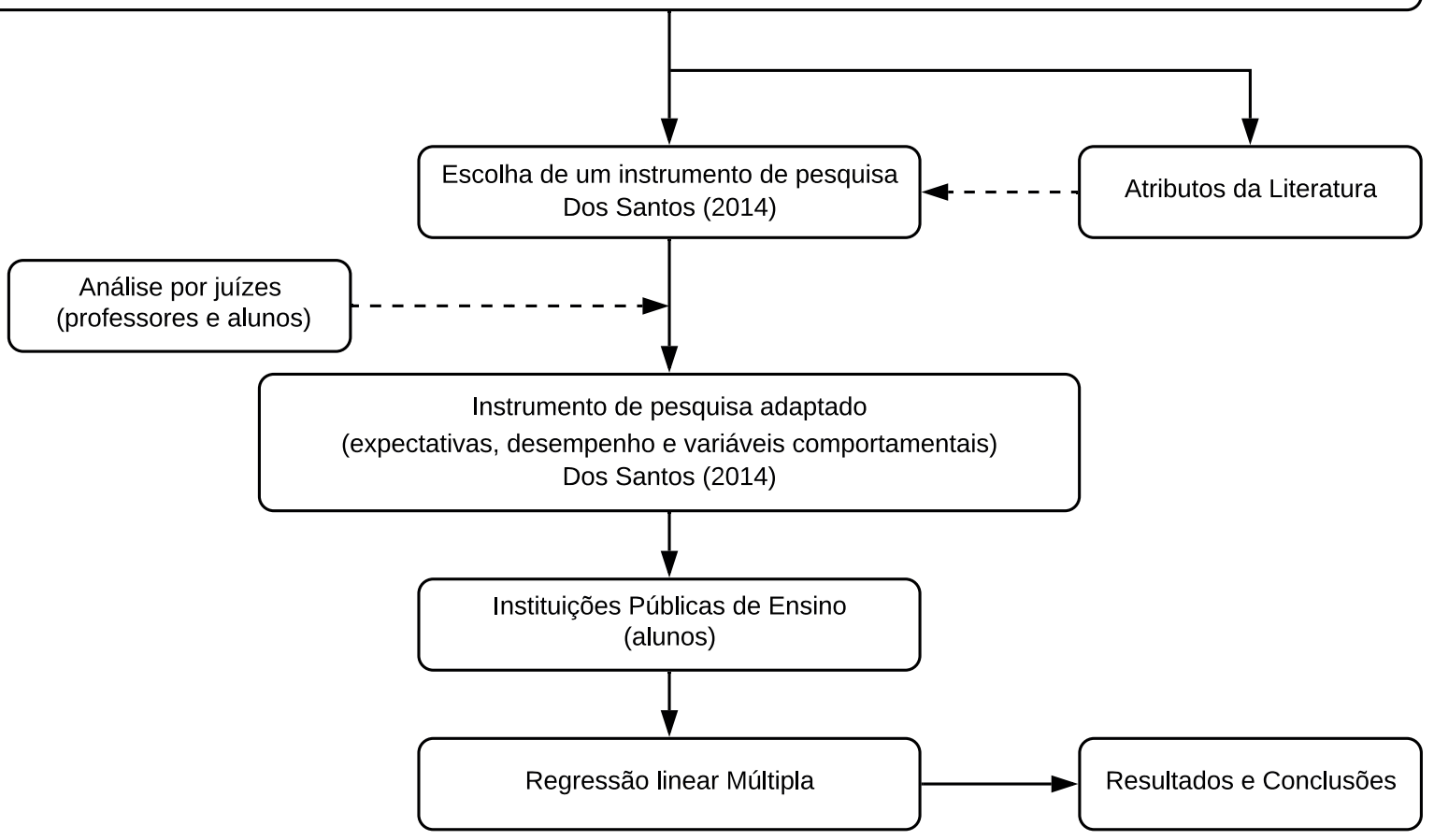

Fonte: Elaborada pelos autores

Um questionário estruturado foi aplicado com 1550 discentes matriculados no $2^{\circ}$ ano do ensino médio de vinte escolas do sertão paraibano. O estudo de Ramos (2015) justifica a escolha por esse público-alvo. Adotando-se um erro de 5\% e um nível de confiança de 95\%, o tamanho amostral alcançou essa 
grandeza. A seleção dos respondentes e instituições obedeceu a critérios probabilísticos (sorteio). Esse procedimento, para Wooldridge (2015), obedece a um dos pressupostos da técnica da regressão linear. Já as amostras foram estratificadas por escola, considerando o número de matriculados em cada uma delas, na série mencionada. Os respondentes foram abordados nos três turnos, em sala de aula, por uma equipe devidamente treinada. No momento da aplicação foram realizados os seguintes procedimentos: apresentação do pesquisador; relato sobre o propósito da pesquisa; sorteio dos alunos que integrariam a pesquisa; leitura em voz alta, por duas vezes, de cada sentença; oferta de tempo para responder cada sentença (aproximadamente 60 segundos) e a prestação de esclarecimentos, quando necessário.

Quanto ao instrumento de pesquisa utilizado, era composto por quatro seções. A primeira contemplou questões demográficas e motivacionais. Nas seções subsequentes, os respondentes foram interrogados sobre o grau de importância e o desempenho percebido da instituição na qual estavam matriculados, considerando atributo a atributo. Para os últimos dois módulos, as respostas foram capturadas mediante utilização de uma escala Likert de 11 pontos, variando de zero (não importante/péssimo desempenho) a dez (muito importante/excelente desempenho). Por fim, o quarto módulo centrou-se em recolher o nível de disposição dos alunos em efetuar rematrícula e em recomendar a organização. Para tanto, a escala Likert de 11 pontos foi empregada, oscilando de zero (sem intenção alguma) a dez (alta intenção). A Figura 3 ilustra as variáveis abordadas nesse estudo. Por findar, uma Análise de Regressão Linear Múltipla (RLM), com o método dos Mínimos Quadrados Ordinários, foi usada com intuito de analisar a relação entre qualidade do serviço, fidelidade dos consumidores e seus comportamentos de recomendar a instituição.
Figura 3 Atributos usados no instrumento de pesquisa

\begin{tabular}{l}
\multicolumn{1}{c}{ Variáveis Independentes $(\mathbf{X})$} \\
\hline V1. Conhecimento prático dos professores \\
\hline V2. Didática dos professores \\
\hline V3. Pontualidade dos professores \\
\hline V4. Estímulo e motivação oferecidos pelo professor ao aluno para \\
a aprendizagem \\
\hline
\end{tabular}

V5. Disponibilidade e qualidade do acervo bibliográfico (quantidade e qualidade)

\begin{abstract}
V6. Qualidade do material didático disponibilizado
V7. Facilidade de acesso e relacionamento com os professores

V8. Horário e dias em que as aulas acontecem

V9. Facilidade de acesso e relacionamento com a direção da escola V10. Acesso à internet através de rede sem fio (wi-fi)

V11. Serviços administrativos disponibilizados aos alunos pela escola (acesso a documentos, resultados de notas, planos de ensino, serviços da biblioteca, requerimentos, entre outros)
\end{abstract}

V12. Cumprimento de prazos administrativos e acadêmicos

V13. Quantidade e qualidade dos recursos multimídia disponíveis em salas de aula

V14. Conforto ambiental da sala de aula (climatização, acústica, iluminação, espaço)

V15. Limpeza e manutenção das salas de aulas, banheiros, corredores e outros espaços

V16. Segurança nas instalações, inclusive no entorno da instituição

V17. Atenção, cordialidade e presteza do pessoal administrativo

V18. Reputação e credibilidade da instituição

Variáveis dummies - feminino: igual a 1 se o aluno é mulher; menor_idade: igual a 1 se o aluno tiver até 18 anos; renda_baixa: igual a 1 se o discente tiver uma renda familiar de até 2 salários mínimos; cidade_polo: igual a 1 se o aluno residir na cidade pólo; motivação: igual a 1 se o aluno escolheu a escola por proximidade com sua residência. Em situações contrárias, os valores assumem a nota 0 .

\section{Variáveis dependentes $(Y)$}

V19. Disposição em rematricular-se nessa escola (fidelidade)

V20. Disposição em recomendar esta escola a outros.

Nota. As variáveis independentes são adaptações daquelas presentes no estudo de Dos Santos (2014). As variáveis dependentes V19 e V20 provêm, respectivamente, dos trabalhos de Ali et al. (2016) e Martínez-Argüelles e Batalla-Bisquets (2016).

Fonte: Elaborada pelos autores 


\section{APRESENTAÇÃO E DISCUSSÃO DE RESULTADOS}

A relação entre qualidade do serviço, fidelidade dos consumidores e seus comportamentos de recomendar a instituição foi avaliada com base no uso do método de Regressão Linear Múltipla. Dois modelos foram propostos nesse estudo. Um regredindo fidelidade (V19) sobre os dezoito atributos de qualidade, e o outro apenas substituindo a variável dependente por disposição em recomendar a escola (V20). O teste de Alfa de Cronbach foi empregado para examinar o nível de confiabilidade de cada um dos construtos. Esse procedimento segue os apontamentos de Hair Junior et al. (2009). Os resultados apontam para níveis expressivos de consistência dos modelos, na ordem de aproximadamente 0,91 para os dois casos.

Ademais, o teste de correlação de Pearson foi usado para verificar o grau de associação entre as variáveis independentes. Os índices oscilaram de 0,206 a 0,608 , indicando que houve a predominância de correlações relativamente baixas entre as variáveis de controle. Por conseguinte, não foi evidenciado dentro do bojo amostral das variáveis explicativas, nem relacionamentos lineares exatos, tampouco valores constantes. Para Wooldridge (2015), esse fenômeno atende a hipótese de colinearidade imperfeita.

\subsection{Atributos da qualidade que influenciam a fidelidade}

A Tabela 1 ilustra o modelo de regressão que associa comportamento de rematrícula dos alunos (variável dependente) aos dezoito atributos de qualidade do serviço (variáveis independentes). Ao nível de significância de $5 \%$, apenas as variáveis didática dos professores (V2), pontualidade dos docentes (V3), fácil acesso e relacionamento com os professores (V7), o atendimento do pessoal administrativo (V17) e imagem corporativa (V18) possuem efeito estatisticamente significativo sobre a intenção de rematrícula na instituição. Esses achados coadunam com as contribuições de Martínez-Argüelles e Batalla-Bisquets (2016) e Ali et al. (2016). No que tange a magnitude, os efeitos foram bem similares $\left(\beta_{\mathrm{V} 2}=0,09\right.$; $\left.\beta_{\mathrm{v} 3}=-0,11 ; \beta_{\mathrm{v} 7}=0,06 ; \beta_{\mathrm{v} 17}=0,08 ; \beta_{\mathrm{v} 18}=0,12\right)$. Contudo, a pontualidade dos docentes (V3) mostrou uma relação negativa. Nesse caso, há de se esperar que menores déficits de qualidade $\left(\mathrm{X}_{\mathrm{V} 2}<0\right)$ no atributo V2 conduzam a maiores níveis de lealdade dos alunos. Para este estudo, essa é a interpretação válida dos parâmetros de inclinação negativos, pois a qualidade percebida em cada atributo foi capturada pela diferença entre os escores de desempenho e de importância atribuída (expectativas). Por sua vez, os coeficientes positivos simbolizam que ampliar o excedente de qualidade do atributo $(X>0)$ aumenta a fidelidade dos consumidores.

Não obstante à insignificância estatística individual (teste $\mathrm{t}$ ) das demais variáveis, o teste $\mathrm{F}$ rejeitou a hipótese de que todos os atributos de qualidade incluídos no modelo não têm efeito sobre o nível de fidelidade (20,98 a 0,0000 de significância). Segundo Wooldridge (2015), nessas condições, há indícios estatísticos de que as variáveis de controle influenciam a variável de resposta. Ao avaliar o $\mathrm{R}^{2}$ ajustado, é possível verificar que $18,9 \%$ da variação de fidelidade é explicada pelos atributos de qualidade incluídos no modelo.

A seguir, os demais pressupostos da regressão linear foram examinados para que o modelo tornasse válido. Quanto à ausência de multicolinearidade, foi desenvolvido o teste do fator de inflação da variância (VIF). Os valores variaram de 1,38 a 2,17 correspondendo a patamares aceitáveis de multicolinearidade. Hair Junior et al. (2009) asseguram que escores próximos a um retratam baixos níveis de colinearidade. Isso ratifica a não violação deste modelo a hipótese da colinearidade não perfeita.

No tocante à hipótese da homocedasticidade dos resíduos, o teste de White foi empregado. $\mathrm{O}$ p-valor $\left(8,4 \mathrm{e}^{-19}\right)$ do teste indica que a hipótese nula (homocedasticidade) é rejeitada ao nível de $1 \%$ de significância. Assim, ocorreu a violação de um dos pressupostos da regressão linear, o qual impossibilita o uso das estatísticas t e F para proceder inferências. Nessas ocorrências, Wooldridge (2015) recomenda o uso do modelo de regressão robusto para retificar o problema. Contudo, esta técnica é válida tão somente para grandes amostras, a exemplo deste estudo $(\mathrm{n}=$ 1550). Após a execução dos procedimentos robustos, a variável intitulada fácil acesso e relacionamento com os professores (V7) não mais apresentou um efeito estatisticamente significativo ao nível de 5\%. Por 
findar, foi avaliada a normalidade dos resíduos, pois é uma condição necessária para empregar os testes t e F. Para tanto, aplicou-se o teste de Shapiro-Wilks que assume como hipótese nula a distribuição normal dos resíduos. O teste proporcionou um p-valor de 0,903. Isso confirma a hipótese da normalidade dos resíduos.

Tabela 1 Modelo de regressão linear entre fidelidade (Y) e qualidade do serviço (X)

\begin{tabular}{|c|c|c|}
\hline Variáveis Independentes & $\begin{array}{c}\text { Modelo } 1 \\
\beta \text { (erro-padrão) }\end{array}$ & $\begin{array}{l}\text { Modelo } 2 \\
\text { (Robusto) }\end{array}$ \\
\hline V2. Didática dos professores & $0,099^{*}(0,035)$ & $0,099^{* * *}(0,042)$ \\
\hline V3. Pontualidade dos professores & $-0,118^{*}(0,029)$ & $-0,118^{*}(0,034)$ \\
\hline V7. Facilidade de acesso e relacionamento com os professores & $0,068^{* * *}(0,03)$ & NS \\
\hline V17. Atenção, cordialidade e presteza do pessoal adm. & $0,080^{* *}(0,03)$ & $0,080^{* * *}(0,03)$ \\
\hline V18. Reputação e credibilidade da instituição & $0,126^{*}(0,03)$ & $0,126^{*}(0,03)$ \\
\hline$R^{2}$ & $19,84 \%$ & $19,84 \%$ \\
\hline$R^{2}$ ajustado & $18,90 \%$ & - \\
\hline Estatística F & $20,98^{*}$ & $15,68^{*}$ \\
\hline Observações & 1550 & 1550 \\
\hline \multicolumn{3}{|l|}{ Variável Dependente: Fidelidade (V19) } \\
\hline
\end{tabular}

Nota (") Significante ao nível de 1\%; ("*)Significante ao nível de $5 \%$.

Legenda NS - não significativo ao nível de $5 \%$. $\beta_{\mathrm{v} 7}=0,068(0,041)$.

Fonte: Pesquisa (2017)

Tabela 2 Modelo de regressão linear entre disposição em recomendar $(\mathrm{Y})$ e qualidade do serviço $(\mathrm{X})$

\begin{tabular}{|c|c|c|}
\hline Variáveis Independentes & $\begin{array}{c}\text { Modelo } 1 \\
\beta \text { (erro-padrão) }\end{array}$ & $\begin{array}{l}\text { Modelo } 2 \\
\text { (Robusto) }\end{array}$ \\
\hline V3. Pontualidade dos professores & $-0,062^{* * *}(0,029)$ & NS \\
\hline V6. Qualidade do material didático disponibilizado & $0,067^{* *}(0,027)$ & $0,067^{* *}(0,029)$ \\
\hline V11. Serviços administrativos & $0,052^{* *}(0,026)$ & NS \\
\hline V12. Cumprimento de prazos & $0,055^{* *}(0,027)$ & NS \\
\hline V17. Atenção, cordialidade e presteza do pessoal adm. & $0,108^{*}(0,033)$ & $0,108^{*}(0,038)$ \\
\hline V18. Reputação e credibilidade da instituição & $0,140^{*}(0,030)$ & $0,140^{*}(0,037)$ \\
\hline $\mathrm{R}^{2}$ & $15,82 \%$ & $15,82 \%$ \\
\hline$R^{2}$ ajustado & $14,83 \%$ & - \\
\hline Estatística F & $15,93^{*}$ & $9,40^{*}$ \\
\hline Observações & 1550 & 1550 \\
\hline
\end{tabular}

Variável Dependente: Disposição em recomendar a escola (V20)

Alfa de Cronbach do modelo: 0,908

Nota $(*)$ Significante ao nível de $1 \% ;(* *)$ Significante ao nível de $5 \%$.

Legenda NS - não significativo ao nível de 5\%. $\beta$ V3 = -0,062 (0,034); $\beta$ V11 = 0,052 (0,027); $\beta$ V12=0,055 (0,029).

Fonte: Pesquisa (2017) 


\subsection{Atributos da qualidade que influenciam a disposição em recomendar a escola}

O segundo modelo regride à disposição em recomendar a escola (variável prevista) sobre os atributos da qualidade do serviço (variáveis preditoras). A Tabela 2 ilustra o resultado dessa regressão linear. Tomando por base um nível de significância de $5 \%$, as variáveis rotuladas qualidade do material didático (V6), atendimento do pessoal administrativo (V17), imagem da organização (V18), pontualidade dos docentes (V3), serviços administrativos (V11) e cumprimento de prazos (V12) foram aquelas que apresentaram efeitos significativos sobre o comportamento dos alunos em proceder um marketing de boca a boca favorável.

No entanto, ao proceder o teste de White ( $p$-valor de $4,4 \mathrm{e}^{-17}$ ) é possível examinar que a hipótese da homocedasticidade é rejeitada ao nível de $1 \%$. Isso implica na necessidade de realizar o modelo de regressão robusto. Os achados dessa nova abordagem apontam para a não significância dos parâmetros de inclinação de V3, V11 e V12. Dessa maneira, somente três variáveis apresentaram efeitos significativos sobre a intenção do alunado em recomendar a instituição de ensino, sendo a imagem corporativa $(\beta=0,140)$ $\mathrm{o}$ atributo que ocasiona maior impacto. A partir do sentido da relação (positiva), pode-se realçar que elevados níveis de qualidade percebida levam a maiores probabilidades dos discentes recomendarem os serviços educacionais a outros sujeitos. Efeito símile também foi constatado no estudo de Martínez-Argüelles e Batalla-Bisquets (2016).

Ao diagnosticar a estatística $\mathrm{F}$ do modelo proposto, nota-se que as variáveis de controle inclusas, em conjunto, são significativas para explicar a disposição em recomendar. Entretanto, muitas delas individualmente não se sustentaram estatisticamente (teste $\mathrm{t}$ ). Esse evento justifica o poder preditivo de $15,82 \%$ do construto.

No mais, os valores do teste do fator de inflação da variância (VIF) permanecem inalterados, pois as variáveis independentes são as mesmas para ambos os modelos. Assim, os níveis de coli- nearidade estão em patamares aceitáveis estatisticamente. Quanto à normalidade dos resíduos, o teste de Shapiro-Wilks com $p$-valor de 0,880 indica que o comportamento normal dos resíduos é evidenciado. Porém, outras arguições postuladas por Wooldridge (2015) ratificam o uso das estatísticas t e F. Para o autor, em grandes amostras, a hipótese da normalidade é dispensável. Essa evidência é intitulada normalidade assintótica. A Figura 4 ilustra os modelos propostos no estudo.

\subsection{Diferenças no impacto da qualidade sobre a fidelidade e a disposição em recomendar, decorrentes das variáveis demográficas e motivacionais}

Para avaliar em que nível os efeitos da qualidade percebida sobre a fidelidade e a disposição em recomendar a escola se diferem em relação aos fatores demográficos e motivacionais dos alunos, foi adicionada a cada regressão anterior variáveis dummies. Consoante Wooldridge (2015), esse artifício captura a diferença de resultado médio entre grupos distintos. A Tabela 3 revela os resultados da regressão linear entre fidelidade e qualidade do serviço somada às variáveis dummies.

Nota-se que os conglomerados formados por faixa etária, renda familiar e a motivação que levou o aluno a optar pela escola veem, de forma distinta, a maneira e intensidade com que a qualidade do serviço influencia a fidelidade dos consumidores. O cluster de alunos com até 18 anos, em relação a outros agrupamentos de idade, percebeu um impacto superior $(\beta=0,428)$ da qualidade do serviço sobre a lealdade. Por outro lado, o grupo composto por alunos com renda familiar de até 2 salários mínimos enxerga uma menor influência $(\beta=-0,276)$ da qualidade do serviço sobre o processo de fidelização; comparando-o com discentes de diferentes faixas de renda. De forma símile, em relação aos demais agrupamentos segregados pela motivação, os alunos que elegeram a escola pela proximidade de suas residências observaram um menor $(\beta=-0,418)$ impacto da qualidade do serviço sobre a fidelidade. 
Figura 4 Modelos propostos a partir de regressão linear robusta

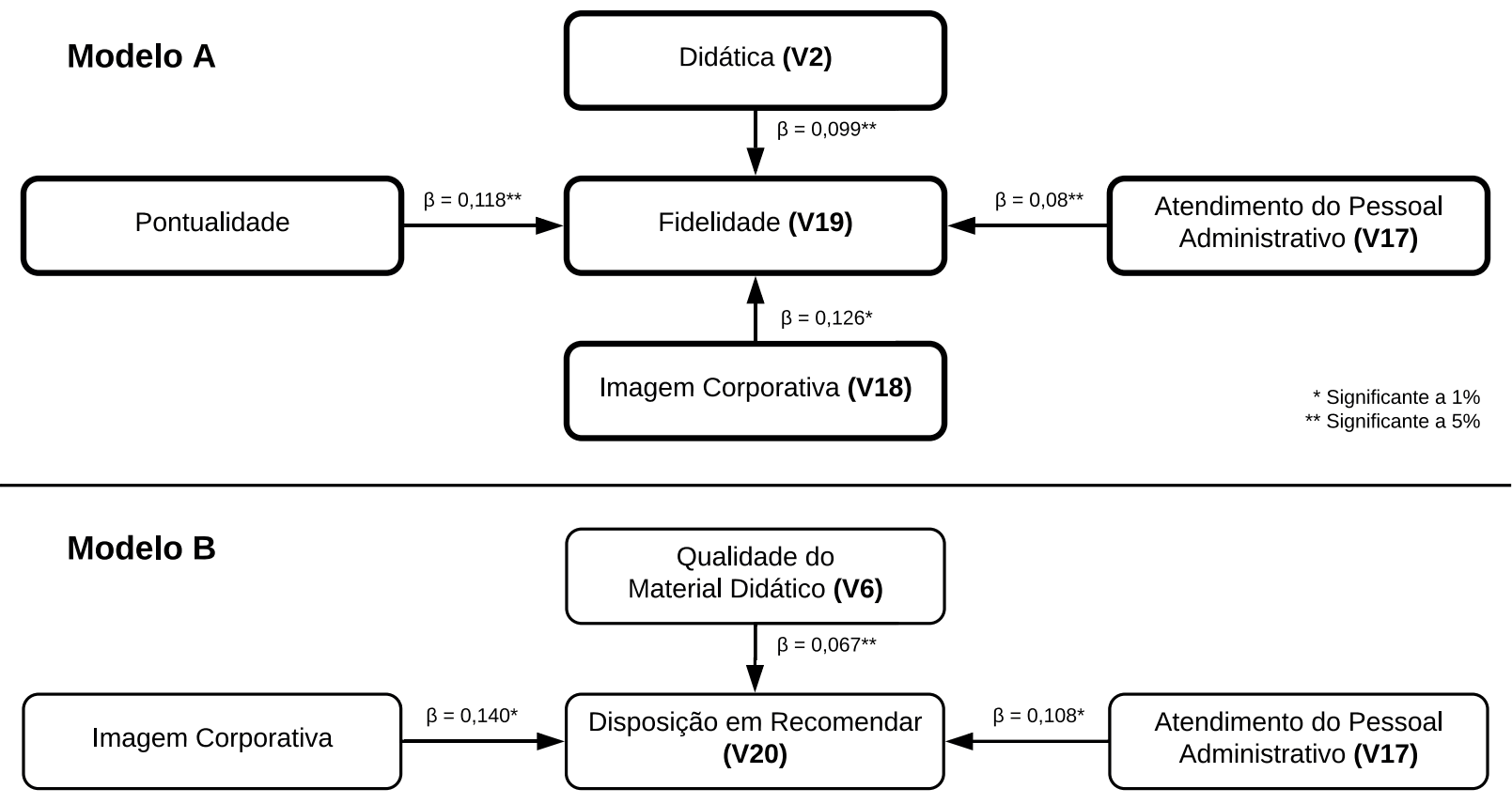

Fonte: Elaborada pelos autores

Tabela 3 Modelo de regressão linear entre fidelidade e qualidade do serviço com variáveis dummies

\begin{tabular}{|c|c|c|}
\hline Variáveis Independentes & $\begin{array}{c}\text { Modelo } 1 \\
\beta \text { (erro-padrão) }\end{array}$ & $\begin{array}{l}\text { Modelo } 2 \text { (Ro- } \\
\text { busto) }\end{array}$ \\
\hline V2. Didática dos professores & $0,102^{*}(0,035)$ & $0,102^{* *}(0,044)$ \\
\hline V3. Pontualidade dos professores & $-0,116^{*}(0,029)$ & $-0,116^{*}(0,034)$ \\
\hline V7. Facilidade de acesso e relacionamento com os professores & $0,078^{* *}(0,030)$ & NS \\
\hline V17. Atenção, cordialidade e presteza do pessoal adm. & $0,085^{* *}(0,033)$ & $0,085^{* *}(0,037)$ \\
\hline V18. Reputação e credibilidade da instituição & $0,117^{*}(0,031)$ & $0,117^{*}(0,038)$ \\
\hline menor_idade (dummy) & $0,428^{*}(0,157)$ & $0,428^{* *}(0,171)$ \\
\hline renda_baixa (dummy) & $-0,276^{* *}(0,128)$ & $-0,276^{* *}(0,126)$ \\
\hline Motivação (dummy) & $-0,418^{*}(0,124)$ & $-0,418^{*}(0,124)$ \\
\hline$R^{2}$ & $21,43 \%$ & $21,43 \%$ \\
\hline$R^{2}$ ajustado & $20,23 \%$ & - \\
\hline Estatística F & $17,97^{*}$ & $14,31^{*}$ \\
\hline Observações & 1550 & 1550 \\
\hline
\end{tabular}

Variável Dependente: Fidelidade (V19)

Nota (") Significante ao nível de 1\%; (") Significante ao nível de $5 \%$. Legenda NS - não significativo ao nível de $5 \%$. $\beta_{v 7}=0,078(0,041)$.

Fonte: Pesquisa (2017) 
Tabela 4 Modelo de regressão linear entre disposição em recomendar e qualidade do serviço com variáveis dummies

\begin{tabular}{l|c|c}
\multicolumn{1}{c|}{ Variáveis Independentes } & $\begin{array}{c}\text { Modelo 1 } \\
\boldsymbol{\beta} \text { (erro-padrão) }\end{array}$ & $\begin{array}{c}\text { Modelo 2 (Ro- } \\
\text { busto) }\end{array}$ \\
\hline V3. Pontualidade dos professores & $-0,061^{* *}(0,028)$ & $\mathrm{NS}$ \\
\hline V6. Qualidade do material didático disponibilizado & $0,060^{* *}(0,027)$ & $0,060^{* *}(0,028)$ \\
\hline V12. Cumprimento de prazos & $0,057^{* *}(0,027)$ & $\mathrm{NS}$ \\
\hline V17. Atenção, cordialidade e presteza do pessoal adm. & $0,111^{*}(0,032)$ & $0,111^{*}(0,037)$ \\
\hline V18. Reputação e credibilidade da instituição & $0,132^{*}(0,030)$ & $0,132^{*}(0,036)$ \\
\hline feminino (dummy) & $0,304^{*}(0,114)$ & $0,304^{*}(0,110)$ \\
\hline menor_idade (dummy) & $0,777^{*}(0,152)$ & $0,777^{*}(0,177)$ \\
\hline$R^{2}$ & $18,05 \%$ & $18,05 \%$ \\
\hline$R^{2}$ ajustado & $16,81 \%$ & - \\
\hline Estatística F & $14,52^{*}$ & $9,55^{*}$ \\
\hline Observações & 1550 & 1550 \\
\hline Variável Dependente: Disposição em recomendar a escola (V20) & & \\
\hline
\end{tabular}

Nota $\left(^{*}\right)$ Significante ao nível de $1 \% ;\left(^{* *}\right)$ Significante ao nível de $5 \%$.

Legenda NS - não significativo ao nível de $5 \%$. $\beta$ V3 $=-0,061(0,033) ; \beta V 12=0,057(0,029)$

Fonte: Pesquisa (2017)

Ademais, esse modelo explica $21,43 \%$ da variação total da fidelidade do alunado. Mesmo que individualmente algumas variáveis independentes inclusas no modelo não tenham se sustentado estatisticamente, é possível afirmar que, em conjunto, elas se mostraram significantes ao nível de $1 \%$ (teste F robusto de 14,31). Quanto à aplicação do teste de White ( $p$-valor de 2,9 $\mathrm{e}^{-20}$ ), diagnosticou-se que a hipótese da homocedasticidade foi rejeitada. Por isso, justifica-se o desenvolvimento de procedimentos robustos à heterocedasticidade. Já os fatores de inflação da variância (VIF), oscilaram de 1,03 a 2,19. Isso reforça que o fenômeno da multicolinearidade encontra-se em patamares aceitáveis.

No que tange ao relacionamento entre disposição em recomendar e qualidade do serviço, os grupos formados por diferentes gêneros e faixas etárias mostraram diferenças significativas (Tabela 4). As mulheres, mais do que os homens, notam que o nível de qualidade ofertada tem um maior impacto $(\beta=0,304)$ sobre a intenção de recomendar por parte do corpo discente. Em relação à faixa etária, contrastando os alunos de até 18 anos com os alunos de idades diferentes, o primeiro grupo entende que o efeito da qualidade sobre o boca a boca positivo é mais intenso $(\beta=0,777)$.

No mais, os índices VIF permanecem inalterados em razão de não modificar as variáveis independentes. Não houve negligência na hipótese da colinearidade imperfeita. Alusivo aos procedimentos robustos, se faz necessário em detrimento a constatação da presença de heterocedasticidade (teste de White com p-valor de 1,6 $\mathrm{e}^{-17}$ ). Notou-se ainda que o modelo robusto foi capaz de explicar $18,05 \%$ da variação da disposição em recomendar a escola.

\section{IMPLICAÇÕES GERENCIAIS}

Entender em que áreas do serviço alocar recursos de forma prioritária é útil e vital para os gestores oxigenarem as unidades de ensino. Ano a ano, o sistema de ensino brasileiro exige dos gestores a habilidade de recrutar alunos, pois é o quantitativo dessa classe que rege o repasse orçamentário. Diante disso, a qualidade do serviço prestado passa a ser uma arma competitiva distinta, conforme pregou Parasuraman, Zeithaml e Berry (1985). Imersos nessa arguição, os achados apontaram o professor e a marca como os 
atributos de relevo. A didática e pontualidade com que os docentes exercem suas atividades, junto com a imagem da escola na sociedade, exercem influência significativa no alcance da fidelidade dos alunos. Optar por maior capacitação dos professores e adoção de estratégia de branding possivelmente tornará os alunos mais fiéis.

Além disso, o branding pode fortalecer a disposição em recomendar a escola para terceiros. Constatou-se que uma melhor reputação do estabelecimento de ensino proporcionaria maior inclinação para boca a boca positivo. Contudo, é salutar que antes de adotar uma ou outra ação de melhoria os gestores avaliem a composição demográfica e comportamental do seu corpo discente. Isso justifica-se em razão de encontrar-se variações no impacto da qualidade do serviço sobre fidelidade e intenções de recomendar, considerando conglomerados de faixa etária, renda familiar, motivação e gênero.

\section{CONSIDERAÇÕES FINAIS}

Este artigo propôs uma discussão sobre quais atributos da qualidade impactam a fidelidade dos discentes e seus comportamentos em recomendar a instituição de ensino, e em que medida esses efeitos diferem tomando por base as variáveis demográficas e motivacionais dos alunos. Para isto, um ensaio teórico-empírico foi desenvolvido com 1550 alunos do ensino médio, regularmente matriculados em vinte escolas do sertão paraibano. Foram capturadas as expectativas dos discentes e suas percepções de desempenho em relação a 18 atributos, bem como as intenções de rematrícula (lealdade) e de recomendação a escola para outros. Foi empregado a regressão linear múltipla, com procedimentos robustos, em razão das evidências estatísticas de heterocedasticidade dos resíduos.

Os resultados revelaram que quatro atributos (didática e pontualidade dos docentes, atendimento do pessoal administrativo e imagem da organização) apresentaram efeito significativo sobre a fidelidade dos consumidores. De maneira análoga, os dois últimos atributos (V17 e V18) também afetaram a intenção de recomendar a organização, juntamente com a qualidade do material didático. Esses achados corroboram os apontamentos de Martínez-Argüelles e Batalla-Bisquets (2016). No mais, a imagem organizacional constitui-se como um dos principais direcionadores dos construtos fidelidade e intenções de recomendar. Os maiores impactos foram observados nessa dimensão do serviço. Não obstante às limitadas contribuições teóricas veiculadas na área da educação, os estudos de Chi e Qu (2008), Bosque e Martín (2008) realçaram essas evidências em um outro contexto. Logo, os gestores das escolas devem aperfeiçoar os níveis de serviço com foco nesses itens.

Constatou-se ainda diferenças significativas nos efeitos da qualidade do serviço sobre as variáveis explicadas. Faixa etária, renda familiar, motivação e gênero foram os elementos que contribuíram para esse fenômeno. O conglomerado de alunos com até 18 anos percebe uma maior influência da qualidade do serviço sobre fidelidade e intenções de recomendar, contrapondo-o com outros arranjos de idade. $\mathrm{O}$ cluster daqueles cuja renda familiar é de até 2 salários mínimos notou que o impacto da qualidade sobre a fidelidade encontra-se em um menor patamar, em relação aos grupos de outras faixas de renda. Um comportamento similar ao último também foi evidenciado entre os agrupamentos regidos pela motivação dos alunos em contratarem os serviços da escola. No tocante ao gênero, as mulheres enxergam que o efeito da qualidade do serviço sobre a disposição em recomendar é de maior magnitude, se comparado com as percepções do público masculino.

Por fim, não obstante as limitações deste estudo, o mesmo contribuiu para alargar o debate sobre qualidade do serviço na educação. Contudo, futuras investigações são necessárias para fins de reexaminar os achados. Recomenda-se replicar o estudo em outros níveis de educação e em culturas diferentes. Avaliar a qualidade do serviço a partir de julgamentos de outros stakeholders (gestores, docentes, sociedade civil) pode ser outro caminho a ser explorado por pesquisadores. 


\section{REFERÊNCIAS}

ABDULLAH, F. The development of HEdPERF: a new measuring instrument of service quality for the higher education sector. International Journal of Consumer Studies, v. 6, n. 30, p. 569-581, 2006.

ALCÂNTARA, V. de C.; LUIZ, G. V.; FERREIRA, A. C.; TEODORO, S. A. S. Dimensões e determinantes da satisfação de alunos em uma instituição de ensino superior. Revista Brasileira de Marketing, v. 3, n. 11, p. 193-220, 2012.

ALI, F.; ZHOU, Y.; HUSSAIN, K.; NAIR, P. K.; RAGAVAN, N. A. Does higher education service quality effect student satisfaction, image and loyalty? A study of international students in Malaysian public universities. Quality Assurance in Education, v. 1, n. 24, p. 70-94, 2016.

ANNAMDEVULA, S.; SHEKHAR, R. Development of HiEdQUAL for measuring service quality in Indian higher education sector. International Journal of Innovation, Management and Technology, n. 3, p. 412-416, 2012.

ASHRAF, M. A.; OSMAN, A. Z. R.; RATAN, S. R. A. Determinants of quality education in private universities from student perspectives: a case study in Bangladesh. Quality Assurance in Education, v. 1, n. 24, p. 123-138, 2016.

BOSQUE, I. R.; MARTÍN, H. S. Tourist satisfaction: A cognitive-affective model. Annals of Tourism Research, v. 35, n. 2, p. 551-573, 2008.

BROCHADO, A. Comparing alternative instruments to measure service quality in higher education. Quality Assurance in Education, v. 17, n. 2, p. 174190, 2009.
BUTTA, B. Z.; REHMAN, K. U. A study examining the students satisfaction in higher education. Procedia Social and Behavioral Sciences, n. 2, p. 5446-5450, 2010.

CAMPOS, D. F.; MARTINS, L. D. S.; NETO, M. L. Qualidade dos serviços na educação superior: uma visão comparativa entre dois cursos de graduação. In: SIMPÓSIO DE ADMINISTRAÇÃO DA PRODUÇÃO, LOGÍSTICA E OPERAÇÕES INTERNACIONAIS, 24., 2011, São Paulo, SP, Brasil. Anais eletrônico. Disponível em: <http://www.simpoi.fgvsp.br/>. Acesso em: 04 de março de 2016.

CARDONA, M. M.; BRAVO, J. J. Service quality perceptions in higher education institutions: the case of a colombian university. Estudios gerenciales, n. 28, p. 23-29, 2012.

CHI, C. G. Q.; QU, H. Examining the structural relationships of destination image, tourist satisfaction and destination loyalty: An integrated approach. Tourism Management, n. 29, p. 624-638, 2008.

CUNHA, E. A.; ANDRADE, D. M.; BRANDÃO, M. M. Atributos do serviço educacional na percepção do discente sobre a qualidade. In: SEMINÁRIOS EM ADMINISTRAÇÃO, 8., 2010, São Paulo, SP, Brasil. Anais eletrônicos. Disponível em: $<$ http://www.fucape.br/_public/producao_cientifica/2/Daniel\%20-\%20 SEMEAD.pdf>. Acesso em: 03 de janeiro de 2016.

DESHIELDS JR. W.; KARA, A.; KAYNAK, E. Determinants of business student satisfaction and retention in higher education: applying Herzberg's two factor theory. International Journal of Educational Management, v. 2, n. 19, p. 128-139, 2005.

DOS SANTOS, G. S. Avaliação da qualidade do serviço educacional numa instituição de ensino superior privada: a perspectiva de alunos de graduação em um estudo longitudinal. 2014, 138p. Dissertação (Mestrado) - Universidade Potiguar, Natal, RN, Brasil, 2014. 
DUARTE, L. R.; PIRATELli, C. L. Gestão da qualidade de serviços no ensino superior: aplicação do método HEDPERF. In: SIMPÓSIO DE ADMINISTRAÇÃO DA PRODUÇÃO, LOGÍSTICA E OPERAÇÕES INTERNACIONAIS, 28., 2015, São Paulo, SP, Brasil. Anais eletrônicos. Disponível em: $<$ http://www.simpoi.fgvsp.br/>. Acesso em: 07 de março de 2016.

EBERLE, L. Identificação das dimensões da qualidade em serviços: um estudo aplicado em uma instituição de ensino superior localizada em Caxias do Sul - RS. 2009, 146p. Dissertação (Mestrado) Universidade de Caxias do Sul, Caxias do Sul, RS, Brasil, 2009.

FITZSIMMONS, J. A.; FITZSIMMONS, M. J. Administração de serviços: operações, estratégia e tecnologia da informação. 4.Ed. Porto Alegre: Bookman, 2005.

GRÖNROOS, C. A service quality model and its marketing implications. European Journal of Marketing, v. 4, n. 18, p. 36-44, 1984.

HAIR JUNIOR, J. F.; BLACK, W.C.; ANDERSON, R.E.; TATHAM, R. L. Análise multivariada de dados. Porto Alegre: Bookman, 2009.

KHOO, S.; HA, H.; MCGREGOR, S. L.T. Service quality and student/customer satisfaction in the private tertiary education sector in Singapore. International Journal of Educational Management, v. 31, n. 4, p.430-444, 2017.

INEP, Instituto Nacional de Estudos e Pesquisas Educacionais Anísio Teixeira. Censo escolar 2016: notas estatísticas. Disponível em: $<$ http://portal. inep.gov.br/ censo-escolar $>$. Acesso em: 01 de maio de 2017.

LANKARA, V. P. D.; YE, Y. A comparative study on students' satisfaction between naung taung monastic high school and kyauk ta lone public high school in southern shan state, Myanmar. Sholar, v. 1, n. 7, p. 277-288, 2015.
LOURENÇO, C. D. da S.; KNOP, M. F. T.; OLIVEIRA, V. C. da S.; SILVA, M. R. J. D. Ensino superior em Administração e percepção da qualidade de serviços: uma aplicação da escala SERVQUAL. In: ENCONTRO DA ANPAD, 15., 2006, Salvador, BA, Brasil, 2006. Anais. Salvador: EnANPAD, 2006. CD-ROM.

MARTÍNEZ-ARGÜELLES, M. J.; BATALLABUSQUETS, J. M. Perceived service quality and student loyalty in an online unuversity. International Review of Research in Open and Distributed Learning, v. 17, n. 4, p. 264-279, 2016.

MILAN, G. S.; CORSO, A.; EBERLE, L. Atributos e dimensões da qualidade em serviços na percepção de alunos de uma IES. In: SIMPÓSIO DE ADMINISTRAÇÃO DA PRODUÇÃO, LOGÍSTICA E OPERAÇÕES INTERNACIONAIS, 16., 2013, São Paulo, SP, Brasil. Anais eletrônicos. Disponível em: $<$ http://www.simpoi.fgvsp.br/>. Acesso em: 03 de março de 2016.

MOSTAFA, M. M. A Comparison of SERVQUAL and I-P Analysis: Measuring and Improving Service Quality in Egyptian Private Universities. Journal of Marketing for Higher Education, n. 16, p. 83 -104, 2007.

NAVARRO, M. M.; IGLESIAS, P. M.; TORRES, R. P. A New Management Element for Universities: Satisfaction with the offered courses. International Journal of Educational Management, v. 6, n. 19, p. 505-526, 2005.

NYAGOWAA, H. O.; OCHOLLA, D. N.; MUTULA, S. M. Service quality, user satisfaction and net-benefits' effect on e-School success: The case of NEPAD's e-School pilot in Kenya. Education for Information, n. 30, p. 93-115, 2013.

PARASURAMAN, A.; ZEITHAML, V. A.; BERRY, L. L. A conceptual model of service quality and its implications for future research. Journal of Marketing, n. 49, p. 41-50, 1985. 
PEREIRA FILHO, E.; TENÓRIO, F. A. G.; SILVA, J. R. Improvement Opportunities: Quality Service in Public Schools. Revista Iberoamericana sobre Calidad, Eficacia y Cambio en Educación, v. 15, n. 2, p. 31-51, 2017.

RAMOS, B. S. Percepção de qualidade das escolas do ponto de vista de alunos do ensino médio. 2015, Dissertação (Mestrado) - Fundação Getúlio Vargas, Rio de Janeiro, RJ, Brasil, 2015.

RAMSEOOK-MUNHURRUN, P.; NUNDLALL, P. Service quality measurement for secondary school setting. Quality Assurance in Education, v. 4, n. 21, p. 387-401, 2013.

SWEIS, R.; DIAB, H.; SALEH, F. I. M.; SUIFAN, T.; DAHIYAT, S. E. Assessing service quality in secondary schools: the case of Jordan. Benchmarking: An International Journal, v. 23, n. 5, p. 207-226, 2016.

SOUZA, C. C. M.; DA SILVA, D.; MORETTI, S. L. A.; GARCIA, M. N. Construção e validação de escala de qualidade de ensino e serviços: um estudo com alunos de cursos de graduação em administração de empresas. REGE, v. 1, n. 21, p. 65-82, 2014.

VERGARA, J.; QUESADA, V. Análisis de la calidad en el servicio y satisfacción de los estudiantes de Ciencias Económicas de la Universidad de Cartagena mediante un modelo de ecuaciones estructurales. Revista Electrónica de Investigación Educativa, n. 13, p. 108-122, 2011.

WOOLDRIDGE, J. M. Introdução à econometria: uma abordagem moderna. São Paulo: Cengage Learning, 2015. 